COMPUTER METHODS IN APPLIED MECHANICS AND ENGINEERING 37 (1983) 109-123

NORTH-HOLLAND PUBLISHING COMPANY

\title{
REMARKS ON 4CST-ELEMENTS FOR INCOMPRESSIBLE MATERIALS*
}

\author{
Noboru KIKUCHI \\ Department of Mechanical Engineering and Applied Mechanics, University of Michigan, Ann Arbor, MI, \\ U.S.A.
}

Received 1 June 1982

\begin{abstract}
Three-node triangular elements obtained from the intersecting diagonals of quadrilaterals are thoroughly investigated for the penalty method formulation of incompressible problems such as Stokes flow, Navier-Stokes flow, and small-deformation rubber elasticity. Equivalence of the present formulation to the one using 4-node quadrilateral isoparametric elements with selective reduced integration, is presented. Convergence, instability phenomenon, smoothing of the pressure distribution are studied together with decompositions into triangular elements.
\end{abstract}

\section{Introduction}

It is well known that the three-node triangular elements, obtained from the diagonal meet of quadrilaterals, are applicable to problems in which the incompressibility of materials is treated as a constraint, by using the exterior penalty method as described in [1]. Because of the characteristic that a quadrilateral is divided into four constant strain triangular elements by intersecting diagonals, such triangular elements are called the 4CST elements in this article. We shall here study details of the 4CST elements in connection with the four-node quadrilateral isoparametric element and the selective reduced integration technique to avoid the phenomenon of locking on to the approximate solution by finite element methods.

Since the work by Zienkiewicz [2], exterior penalty methods have been widely applied to solve the constrained problems such as Stokes/Navier-Stokes flow problems which are restricted by the divergence free condition $\operatorname{div} \boldsymbol{u}=0$, while a similar approach was introduced to study a plate bending problem by Zienkiewicz, Taylor and Too [3]. The technique of reduced or selective reduced integration was essential to the success of quadrilateral isoparametric elements for the incompressible problem, although such a technique is not necessary for the family of triangular elements.

Clear understanding of the selective reduced integration penalty method was obtained by Malkus and Hughes [4] in the course of studying its equivalence to a mixed finite element method, and by Reddy [5], Bercovier [6], Oden and Kikuchi [7] for the study of convergence of the penalty method. The method was also advocated by Lee, Gresho, and Sani [8] for its practicability. An important feature of that work is the introduction of the smoothing scheme of the pressure identified by the penalty method.

*This was presented at the SECTAM XI at Huntsville, Alabama, April 8-9, 1982.

0045-7825/83/0000-0000/\$03.00 (C) 1983 North-Holland 


\section{Penalty formulation to the Stokes flow problem}

We shall first consider the Stokes flow problem in order to emphasize the penalty resolution rather than other complications in the formulation, since extension of the results for the penalty method to other nonlinear problems is straightforward provided they are well posed.

Let $\boldsymbol{u}$ be a velocity field of the Stokes flow, and let $\boldsymbol{f}$ denote the body forces that may be constructed by the non-homogencous velocity boundary conditions. If the incompressibility of the fluid flow is represented by the divergence free condition div $\boldsymbol{u}=0$, where div is the divergence operator, the problem is defined by the virtual work principle: find

$$
\begin{aligned}
& \boldsymbol{u} \in K: \quad a(\boldsymbol{u}, \boldsymbol{v})=f(\boldsymbol{v}), \quad \forall \boldsymbol{v} \in K \\
& K=\left\{\boldsymbol{v} \in \boldsymbol{H}_{0}^{1}(\Omega): \operatorname{div} \boldsymbol{v}=0 \text { in } \Omega\right\}
\end{aligned}
$$

and where $\boldsymbol{H}_{0}^{1}(\Omega)$ is the Sobolev space obtained by the completion of the test function space $C_{0}^{\infty}(\Omega)$ with respect to the Sobolev norm: $\|\boldsymbol{v}\|_{1}=\left\{\left(v_{i, j}, v_{i, j}\right)+\left(v_{i}, v_{i}\right)\right\}^{1 / 2}$ with $v_{i, j}=\partial v_{i} / \partial x_{j}$. The bracket $(\cdot, \cdot)$ is the $L^{2}$-inner product on a Lipschitz domain $\Omega$ in $\mathbb{R}^{2}$, while

$$
a(\boldsymbol{u}, \boldsymbol{v})=\int_{\Omega} \mu u_{i, j} v_{i, j} \mathrm{~d} \Omega \quad \text { and } \quad f(\boldsymbol{v})=\int_{\Omega} f_{i} v_{i} \mathrm{~d} \Omega
$$

Here $\mu$ is the viscosity with bounds

$$
\mu_{0} \leq \mu(x) \leq \mu_{1}, \quad \forall x \in \Omega
$$

It is known that the problem has a unique solution $\boldsymbol{u}$ which is moreover, smooth provided the domain $\Omega$ and the body forces $f$ are smooth enough, see for example, [9]. One of the difficulties arising in the finite element approximation of the Stokes problem (1) is that an adequate approximation of the velocity field satisfying the constraint $\operatorname{div} v=0$ may only be possible in certain special cases. As shown in [10, p. 77-79], given a uniform mesh to a rectangular domain, it is possible to construct an interpolation operator from $K$ into $K_{h}$ provided that mesh arises from the partition of a rectangle into four triangles. Furthermore, error bounds the approximation $\boldsymbol{u}_{h}$ can be established to be

$$
\left\|\boldsymbol{u}-\boldsymbol{u}_{\boldsymbol{h}}\right\|_{1} \leq C h^{s-1}\|\boldsymbol{u}\|_{s}
$$

where $s(1<s<2)$ parametrises the smoothness of the solution to the problem $(1)$.

However, it is not known whether a special interpolation operator from $K$ into $K_{h}$ is constructible for a non-uniform mesh in a non-rectangular domain. Then the crror estimate (2) does not have any meaning. Moreover, an operator, so constructed, is not usable in the ordinary way.

A method of resolving the constraint $\operatorname{div} \boldsymbol{u}=0$ is the so-called exterior penalty method, originally introduced by Courant [15] and mathematically studied by Aubin [11] and Lions [12]. Introducing a penalty parameter, $E>0$, we modify the problem (1) to one of finding 


$$
u_{E} \in V: \quad a\left(u_{E}, v\right)+1 / E\left(\operatorname{div} u_{E}, \operatorname{div} v\right)=f(v), \quad \forall v \in V
$$

where $V$ is $\boldsymbol{H}_{0}^{1}(\Omega)$. It is shown in [9] that if $E$ goes to zero, the approximate solution $u_{E}$ converges to the solution $\boldsymbol{u}$ of (1). Moreover, the hydrostatic pressure $p_{E}$, identified by

$$
p_{E}=-\frac{1}{E} \operatorname{div} u_{E}
$$

converges to the true hydrostatic pressure $P$ as $E$ goes to zero.

As shown in [7], the rate of convergence to $u$ is indicated by

$$
\left\|\boldsymbol{u}-\boldsymbol{u}_{E}\right\|_{1}+\| p-p_{E} \rrbracket_{0} \leq C \sqrt{E},
$$

since the pressure $p$ is uniquely determined only to within a constant function on $\Omega$. Here the norm $\| \cdot \prod_{0}$ is any norm equivalent to the quotient norm of $L^{2}(\Omega)$ with respect to the set of constant functions forming the kernel of the transpose of the divergent operator. If the kernel is a singleton, the bound (5) can be improved from $C \sqrt{E}$ to $C E$.

\section{Finite element approximations}

Let the domain $\Omega$ in the two-dimensional field be polygonal, that is, covered by 4 CST elements obtained by dividing each quadrilateral into four triangles using its diagonals, as shown in [1]. Let $V_{h}$ be the finitc element approximation of the Sobolev space

$$
V_{h}=\left\{\boldsymbol{v}_{h} \in C(\bar{\Omega}): \boldsymbol{v}_{h} \in P\left(\Omega_{e}\right) \text { in } \Omega_{e}\right\}
$$

where $C(\bar{\Omega})$ is the set of all continuous functions on $\bar{\Omega}$ and $P\left(\Omega_{e}\right)$ is the set of all piecewise linear polynomials such that

$$
d_{1}+d_{3}=d_{2}+d_{4}
$$

Here $d_{i}=\operatorname{div} v$ on the subtriangle $K_{i}$ of a quadrilateral $\Omega_{e}$ as shown in Fig. 1. A function $v_{h}$ is a linear polynomial on each subtriangle. The special relation (7) can be proved by defining the coordinate system $(s, t)$, the axes of which pass through the diagonals of a quadrilateral as shown in Fig. 2. If the angle $\theta$ is not $\frac{1}{2}$, the coordinate transformation becomes

$$
s=x+y \tan \theta \text { and } t=y / \cos \theta .
$$

Thus the divergence of the velocity is expressed by

$$
\operatorname{div} v=\partial u / \partial x+\partial v / \partial y=\partial u / \partial s+\tan \theta \partial v / \partial s+(1 / \cos \theta) \partial v / \partial t
$$

Since the first derivative $\partial u / \partial s$ has the same value on the 1 st and 4 th subtriangles, and since similar relations hold on two adjacent subtriangles, condition (7) is satisfied. 


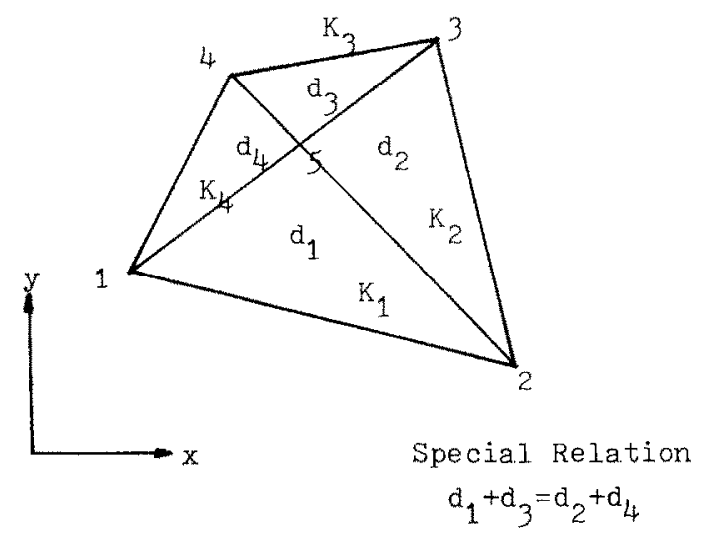

Fig. 1. 4CST-finite elements.

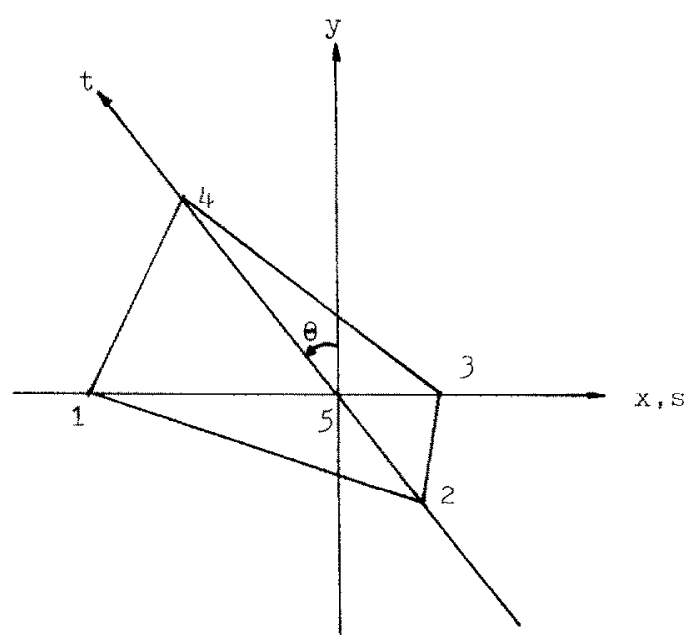

Fig. 2. Coordinate transformation to $(s, t)$.

Because of relation (7), the number of constraints imposed in the finite element model from the incompressibility div $v_{h}=0$, is reduced by one per quadrilateral. More precisely, three constraints are imposed in the finite element approximation if 4CST elements are used, although four constraints are introduced from the four triangles if the relation (6) does not hold. This provides the positive semi-definiteness of the stiffness matrix related to the penalty term in (3). Moreover, if an extra node is arranged within each quadrilateral, more degrees of freedom in the finite clement model are obtained. These yicld unlocked solutions as shown in [1]. Mathematically, the cases of locked and unlocked approximations can be distinguished by the relation

$$
\operatorname{div} \boldsymbol{v}_{h}=0 \text { in } V_{h} \text { implies } \boldsymbol{v}_{h}=\mathbf{0}
$$

If (8) is true, we will have only locked solutions from the finite element approximation of the penalty formulation, since the stiffness matrix due to the penalty becomes positive definite. Indeed, the divergence operator is continuous on $V_{h}$ and

$$
\left(\operatorname{div} v_{h}, \operatorname{div} v_{h}\right)=0 \text { implies } \boldsymbol{v}_{h}=\mathbf{0} .
$$

Because of the finite dimensionality of the space $V_{h}$, it follows that

$$
\left(\operatorname{div} v_{h}, \operatorname{div} v_{h}\right) \geq c\left\|v_{h}\right\|_{1}
$$

where $c$ is a positive constant dependent upon $V_{h}$. Similarly, it is possible to show that if the number of constraints exceeds the number of degrees of freedom in the model, the estimate (9) is also obtained by checking the rank condition of the matrix related to the penalty.

It is noted that if boundary conditions other than those of the Dirichlet type are imposed, locked solutions may not have zero values in the domain. However, these can be easily recognized since they are physically unreasonable. 
For the unlocked case, since the relation

$$
\left(\operatorname{div} \boldsymbol{u}_{E h}, \operatorname{div} \boldsymbol{u}_{E h}\right) \leq E\left\{a\left(\boldsymbol{u}_{E h}, \boldsymbol{u}_{E h}\right)+f\left(\boldsymbol{u}_{E h}\right)\right\}
$$

holds for the finite element solution $\boldsymbol{u}_{E h}$ of (3), then

$$
\left\|\boldsymbol{u}-\boldsymbol{u}_{E h}\right\|_{1}<c\|\boldsymbol{u}\|_{s}\left\{\sqrt{E}+h^{2-1}\right\},
$$

for the uniform meshes for a rectangular domain $\Omega$, as shown by Mercier [13]. In that work it is also suggested that the estimate (10) might hold for all practical purposes even for non-uniform meshes. That is, the penalty finite element approximation $u_{E h}$ by 4CST elements converges to the solution of the Stokes flow problem as the penalty parameter $E$ and the mesh size $h$ go to zero.

The above discussion, however, does not imply anything about the hydrostatic pressure, a quantity of major interest in solving the problem.

\section{Approximation of the hydrostatic pressure}

One natural choice of the definition of hydrostatic pressure in the finite element analysis is the same form, (4), as in the continuous case, i.e., $P_{E h}=-\operatorname{div} \boldsymbol{u}_{E h} / E$. However, this choice might not be adequate, since the special relation (7) yields the condition

$$
p^{1}+p^{3}=p^{2}+p^{4}
$$

where $p^{i}=-\operatorname{div} u_{E n} / E$ in the subtriangle $K_{i}$ of an element $\Omega_{e}$. It is common to identify the pressure with its average value on each quadrilateral consisting of four triangular elements. In this article, we shall take the weighted average value

$$
p_{\text {Eh }}=\left(p^{1} A_{1}+p^{2} A_{2}+p^{3} A_{3}+p^{4} A_{4}\right) / A
$$

in order to form the corresponding mixed finite element formulation. Here, $A_{i}$ is the area of the subtriangle $K_{i}$ and $A$ is the area of a quadrilateral. If the mesh is uniform, this corresponds to the case of taking the average. For the weighted average pressure $P_{E h}$, the penalty formulation (3) is also expressed in the form

$$
\begin{aligned}
& a\left(u_{E h}, v_{h}\right)-\left(p_{E h}, \operatorname{div} v_{h}\right)=f\left(v_{h}\right), \quad \forall v_{h} \in V_{h} \\
& \left(q_{h}, E p_{E h}+\operatorname{div} u_{E h}\right)=0, \quad \forall q_{h} \in Q_{h}
\end{aligned}
$$

where $Q_{h}$ is a set of piecewise constant functions. More precisely, $q_{h}$ in $Q_{h}$ is a constant function on each 4 CST element $\Omega_{e}$.

Let the set $K_{h}^{*}$ be defined by

$$
K_{h}^{*}=\left\{q_{h} \in Q_{h}:\left(q_{h}, \operatorname{div} v_{h}\right)=0, \forall v_{h} \in V_{h}\right\}
$$


Since the homogeneous Dirichlet boundary condition is assumed, the set $K_{h}^{*}$ is not a singleton. Indeed, the checkerboard pattern of the distribution is in $K_{h}^{*}$ as shown in Fig. 3. This is identical to the case of 4-node quadrilateral isoparametric elements with the one-point Gaussian quadrature rule for the penalty term. That is, the nature of the kernel of the approximate gradient operator $B_{h}^{*}$ defined by

$$
\left(B q_{h}, v_{h}\right)=\left(q_{h}, \operatorname{div} v_{h}\right), \quad \forall q_{h} \in Q_{h}, v_{h} \in V_{h}
$$

for the 4CST element is similar to that for the 4-node isoparametric element with selective reduced integration.

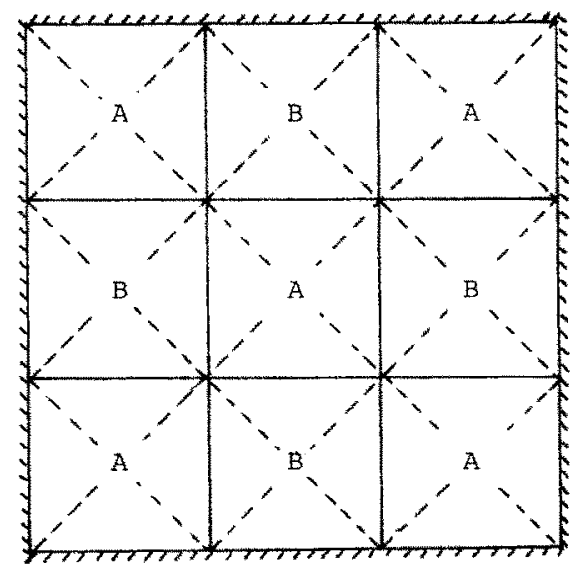

$$
A, B \in R
$$

Fig. 3. Global kernel of the approximate gradient operator for the Dirichlet boundary condition.

PROPOSITION 4.1. If the finite element mesh is uniform in a rectangular domain in $\mathbb{R}^{2}$, the set $K_{h}^{*}$ is not a singleton.

The claim stated above can be verified by considering the two cases of the center nodes in quadrilaterals and of the corner nodes of quadrilaterals. In fact, using the notation described in Fig. 4 , for the $n$th center node the pressure $q_{h}$ must be restricted by

$$
q^{1}=q^{3} \text { and } q^{2}=q^{4}
$$

in order to satisfy the equation

$$
2\left(q_{h}, \operatorname{div} v_{h}\right)=v_{n}^{1}\left(-q^{2}+q^{4}\right)+v_{n}^{2}\left(q^{1}-q^{3}\right)=0, \quad \forall v_{n}^{1}, v_{n}^{2}
$$

within a quadrilateral. Since condition (11) is imposed on the approximate pressure, it follows from (16) that

$$
q^{1}=q^{2}=q^{3}=q^{4}=C .
$$


Center Node

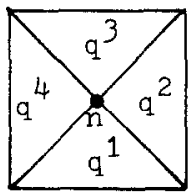

Corner Node

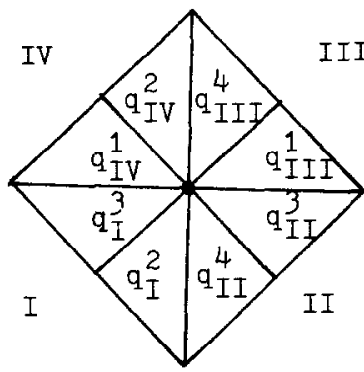

$$
\begin{aligned}
& \mathrm{q}_{I}=\mathrm{q}_{I}^{2}+\mathrm{q}_{I}^{3} \\
& \mathrm{q}_{I I}=\mathrm{q}_{I I}^{3}+\mathrm{q}_{I I}^{4} \\
& \mathrm{q}_{I I I}=\mathrm{q}_{I I I}^{4}+\mathrm{q}_{I I I}^{1} \\
& \mathrm{q}_{I V}=\mathrm{q}_{I V}^{1}+\mathrm{q}_{I V}^{2}
\end{aligned}
$$

Fig. 4. Numbering system for the pressure.

Similarly for the $n$th corner node of a quadrilateral, we have

$$
2\left(q_{h}, \operatorname{div} v_{h}\right)=v_{n}^{1}\left(q_{\mathrm{I}}-q_{\mathrm{II}}-q_{\mathrm{III}}+q_{\mathrm{IV}}\right)+v_{n}^{2}\left(q_{\mathrm{I}}+q_{\mathrm{II}}-q_{\mathrm{III}}-q_{\mathrm{IV}}\right)=0, \quad \forall v_{n}^{1}, v_{n}^{2}
$$

This yields the condition

$$
q_{\mathrm{I}}=q_{\mathrm{III}}=A \quad \text { and } \quad q_{\mathrm{II}}=q_{\mathrm{IV}}=B
$$

for real numbers $A$ and $B$, since (11) is imposed.

Four-node elements with the one-point Gaussian integration have the identical value of the term $\left(q_{h}, \operatorname{div} v_{h}\right)$ as does the 4CST-element. Under the assumption that the pressure field $q_{h}$ is constant on a quadrilateral,

$$
\int_{\Omega_{e}} q_{h} \operatorname{div} v_{h} \mathrm{~d} \Omega=q_{h} \int_{\Omega_{e}} \operatorname{div} v_{h} \mathrm{~d} \Omega=q_{h} \int_{\Gamma_{e}} v_{h} \cdot n \mathrm{~d} \Gamma
$$

where $\Gamma_{e}$ is the boundary of a 4CST element $\Omega_{e}$ and $n$ is the unit vector normal outward to $\Gamma_{e}$. This means that the value of the velocity at the center node cannot contribute to the pressure term if a constant pressure field is assumed on a quadrilateral $\Omega_{e}$. Furthermore, the expression (19) is exactly the same as in the case of a 4-node isoparametric element which uses the one-point Gaussian integration scheme. This shows the following.

PROPOSITION 4.2. The 4CST element is equivalent to the 4-node quadrilateral isoparametric element with the one-point integration scheme, as far as the pressure term $\left(q_{h}\right.$, div $\left.v_{h}\right)$ is concerned. 
In above, we have shown that the 4CST element provides characteristics in the kernel of the approximate gradient operator and a value of the term $\left(q_{h}\right.$, div $\left.v_{h}\right)$ similar to the most popular penalty method, using the 4-node isoparametric element with one-point Gaussian selective reduced integration. However, this does not mean that both methods are same. Within a quadrilateral, the 4CST element has 10 degrees of freedom and 3 discrete constraints, while the 4-node element has 8 degrees of freedom and 1 discrete constraint if the selective reduced integration scheme is applied. Thus the ratio of the degrees of freedom to the number of constraints for the 4CST element is smaller than that for the 4-node element.

$\Lambda$ a further remark on Propositions 4.1 and 4.2 , exactly the same conclusions can be drawn for the pressure term $\left(q_{h}\right.$, div $\left.v^{h}\right)$ obtained by the penalty method, namely,

$$
a\left(u_{E h}, v_{h}\right)+\frac{1}{E} \sum_{e=1}^{N} \int_{\Omega_{e}}\left(\left.\frac{1}{A} \sum_{i=1}^{4} \operatorname{div} v_{E h}\right|_{K_{i}} A_{i}\right) \operatorname{div} v_{h} \mathrm{~d} \Omega=f\left(v_{h}\right), \quad \forall v_{h} \in V_{h},
$$

where div $\left.u_{E h}\right|_{K_{i}}$ is the divergence of $u_{E h}$ on the subtriangle $K_{i}$ of $\Omega_{e}$. The penalty in $(20)$ is formed for the weighted average value of the divergence. That is, as $E \rightarrow 0$ we have

$$
\left.\frac{1}{A} \sum_{i=1}^{4} A_{i} \operatorname{div} \boldsymbol{u}_{E h}\right|_{K_{i}} \rightarrow 0
$$

but not each individual div $\left.v_{E h}\right|_{K_{i}} \rightarrow 0$. In this case, the ratio of the degrees of freedom and the number of constraints becomes higher than that of the 4-node element.

\section{Numerical experiments and remarks}

We shall carefully study the 4CST element in comparison with the 4-node isoparametric element with the one point Gaussian integration rule.

The first numerical experiment is the checking of the convergence result $(10)$ of the penalty finite element approximation. To do this, let us consider a rigid punch problem shown in Fig. 5 . Because of the existence of the free boundary, $K_{h}^{*}$ is a singleton, and then the estimate (10). is modified to

$$
\left\|\boldsymbol{u}-\boldsymbol{u}_{E h}\right\|_{1} \leq C\left(\|\boldsymbol{u}\|_{s,}\|p\|_{s-1}\right)\left\{\boldsymbol{E}+h^{s-1}\right\}
$$

Although the problem to be solved belongs to a class of contact problems for solids, the formulation is almost identical to the Stokes flow problem. Suppose that no friction is assumed beneath the rigid punch, the depth of indentation is $d=-0.2$ that is $2 \%$ of the depth of the foundation shown in Fig. 5. Young's modulus is $E=1$, and that Poisson's ratio is $\nu=0.4999$. These correspond to the case of $\mu=\frac{1}{3}$ and $E=6 \times 10^{-4}$ in the Stokes flow problem. Since the contact stress under the flat punch in the semi-infinite domain in $\mathbb{R}^{2}$ has the form $\sigma_{n}=$ $\sigma_{0} / \sqrt{a^{2}-x^{2}}$, the contact stress in the present example is almost a function in $L^{2}(\Gamma)$. Thus, applying the trace theorem, it can be expected that the solution $u$ is in $H^{3 / 2-\delta}(\Omega), \delta>0$. Thus, the regularity parameter $s$ in the estimate (21) is bigger than $\frac{3}{2}-\delta$. The results shown in Fig. 6 indicate that the quality of the approximation is better than those expected from the theory of convergence. 
Depth of Indentation -0.2

Young $s$ Nodulus $E=1$

Poisson's Ratio $v=0.4999$

Frictioniess Contact

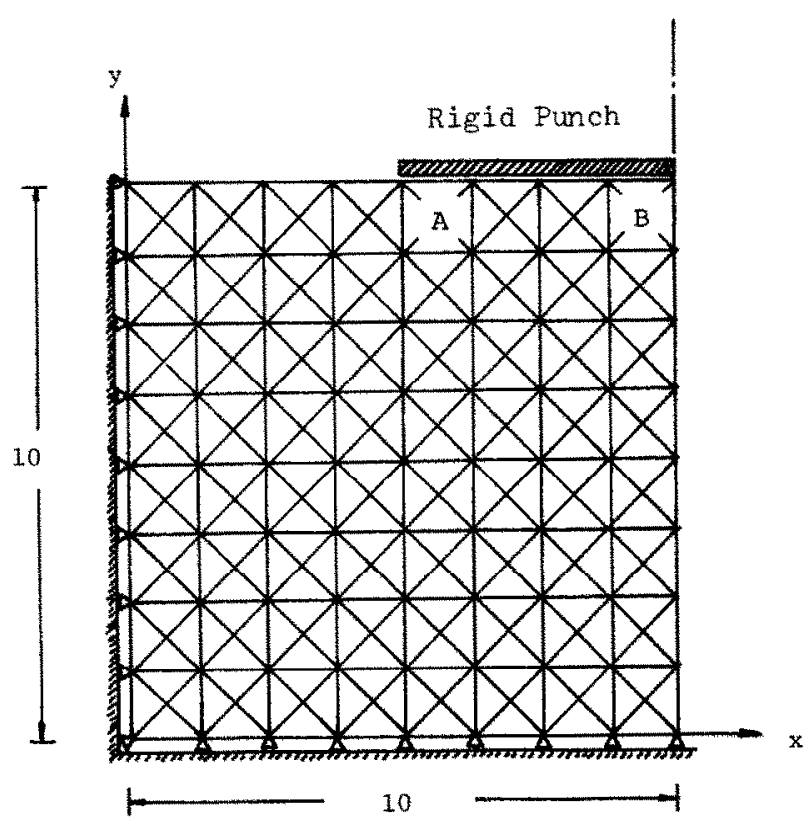

Fig. 5. Finite element model to the indentation problem of a rigid flat punch.

$$
\begin{aligned}
& E_{R}=E_{h}-E_{10 / 8} \\
& h_{R}=h-10 / 8 \\
& E_{h}=\left\{\int_{\Omega} E_{i j} E_{i j} d \Omega\right\}^{1 / 2}
\end{aligned}
$$

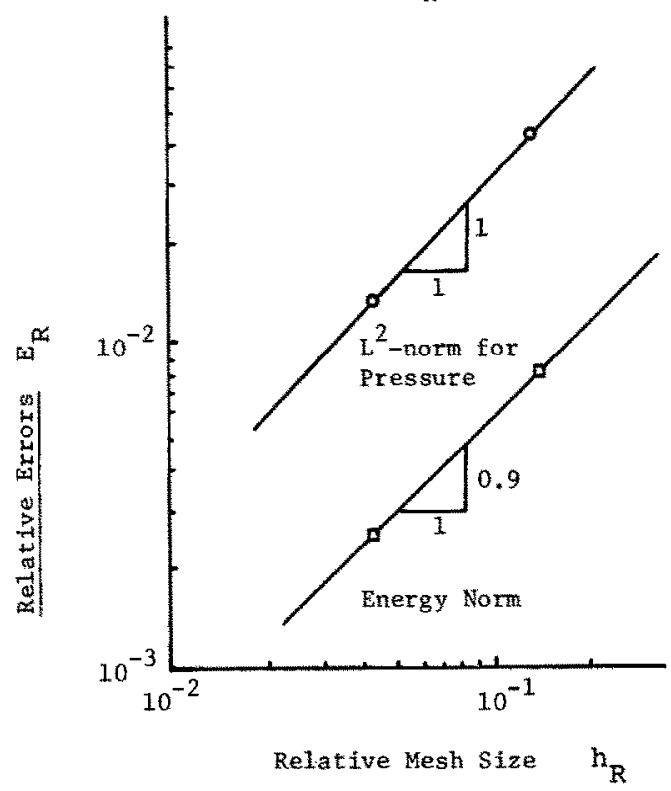

Fig. 6. Convergence of the 4CST-element.

However, it does not necessarily follow that the quality of the approximate solution as perfect. Indeed the pressure computed by (12) has oscillation in its distribution as shown in Fig. 7. Similar behavior is, however, observed for the hydrostatic pressures computed by the 4-node isoparametric element with the selective reduced integration and by the averaged penalty scheme (20). The graphs in Fig. 7 are those of pressure distributions along the line $x=0.625 \mathrm{~m}$ obtained by the three different methods. The three methods provide almost identical results, although the 4-node isoparametric element gives the smallest oscillation.

It is next of interest to check whether or not some unstable mesh patterns are obtained from the continuation of the process of incremental methods for large deformation analysis using similar formulations to (3). This is motivated by the observation of the deformation pattern shown in Fig. 8 for the rigid punch problem. If the deformation (or the velocity field in the flow problem) near to the corner of the fixed and free boundaries is enlarged, it is possible to observe a zig-zag pattern of the deformation. Then it is easy to imagine that if the deformation is continued incrementally, the finite element mesh might have large distortion. We shall check this by solving a large deformation problem for viscoplastic material, the formulation of which is similar to the Stokes problem considered here except for the material constant $\mu$ that depends upon the stress level. Applying an incremental method together with updating the finite element mesh representing the material grid (according to the deformation computed by $\Delta u \times \Delta t$ for the increment of the velocity and the time increment at each nodal point), an 


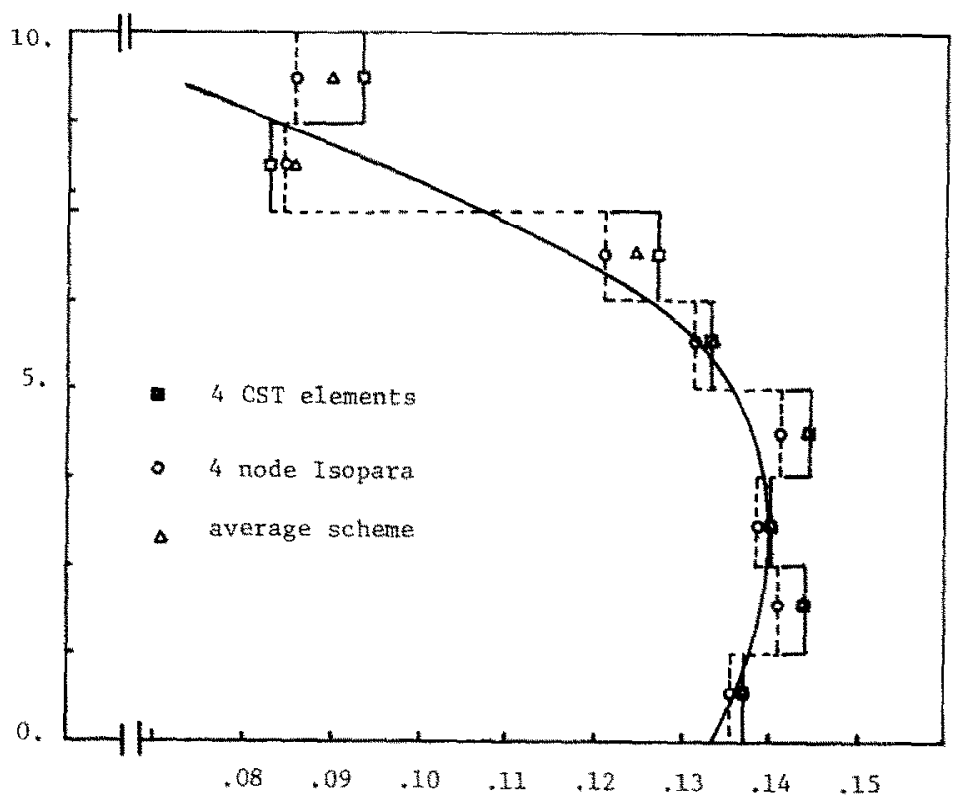

Fig. 7. Pressure distribution along the line $x=0.625 \mathrm{~m}$.

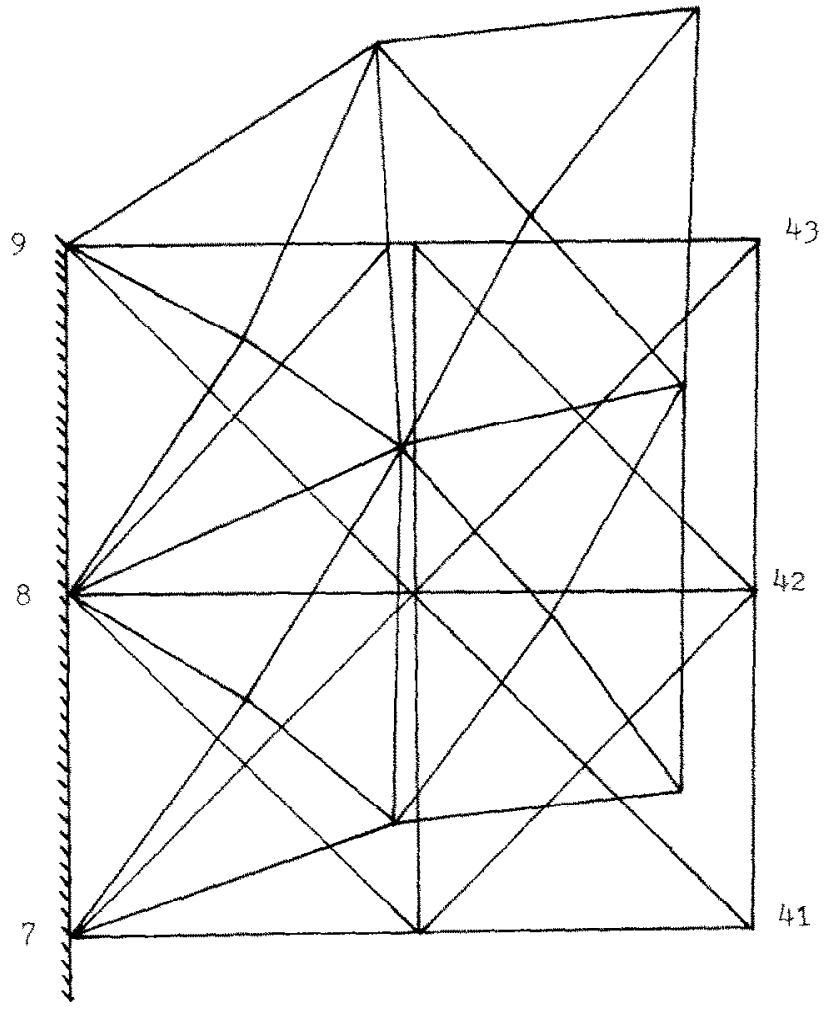

Fig. 8. Irregular deformation at the corner of the rectangular domain (displacements are enlarged tenfold).

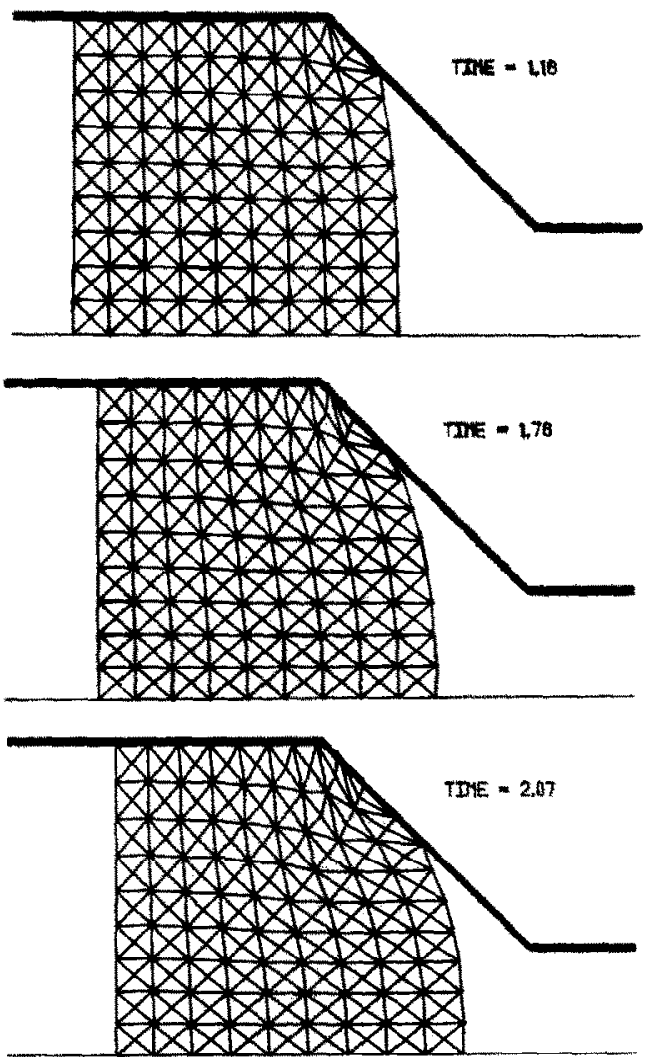

Fig. 9. Extrusion problem for viscoplastic materials associated with Von Mises type flow rule. 
extrusion problem is solved as shown in Fig. 9. It is clear that if the ratio of the sizes of entrance and exit are large, the finite element mesh undergoes a zigzag distortion near the ramp, as several quadrilaterals degenerate to triangles along its slope. The ripple on the deformation is reduced by refining finite element meshes, but cannot be completely eliminated. It is unclear how the distortion of the material deformation affects the value of the stress and thence the yielding of the material. Similar unstable deformation occurs even in the case that the body is in a field of almost uniform tension. If the cold-forming process governed by the elasto-plasticity is involved instead of the visco-plasticity with the incompressibility constraint, unstable deformations can be reduced considerably because of compressibility of elastic strain. Another factor contributing to instability is the boundary condition. If there are too many fixed boundary conditions and too many intersections of fixed and free boundary, then the amount of oscillation increases in both the deformation and the pressure.

Let us now look at the pressure field in a 4CST-element. Fig. 10 shows the pressure distribution in two 4CST-elements which are located under the rigid punch. Element $\mathrm{A}$ is at the edge of the punch, and element B is at the center of the punch. It is clear that the pressure in each subtriangle is quite different from the average value in element $A$, while element $B$ has no variation in its four subtriangles. From this, however, the question arises as to whether the average value of the pressure should be used to check the yielding of the material when the elasto- or visco-plasticity are considered. If the yielding is checked on each triangle, and if the finite element mesh is not updated, there is still the pressure constraint (11) to be satisfied by the finite element solutions. In general, the yielding is described on each subtriangle without using the average value of the pressure for the 4 CST element. This is the main difference from the 4-node isoparametric element with the reduced integration scheme in which the stresses are accurately evaluated only at those integration points which are reduced. Thus, the yielding is checked only on each quadrilateral. It is noted that if the elasto-plasticity is considered in the rigid punch problem, the lower triangular part of element $\mathrm{A}$ becomes plastic at first. If a 4CST-element scheme is used with individual checking of yielding, it is possible to treat the development of the plasticity. However, if the 4-node isoparametric element with the reduced integration is used to solve the same elasto-plastic punch problem, numerical results may be less good. In order to obtain that the lower triangular part is plastic, an 8-node or 9-node isoparametric element at least, with the reduced integration scheme must be applied when quadrilateral elements are chosen.

The next study is related to the process of updating the finite element mesh for large-

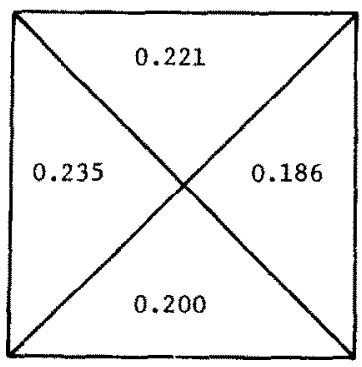

Element A

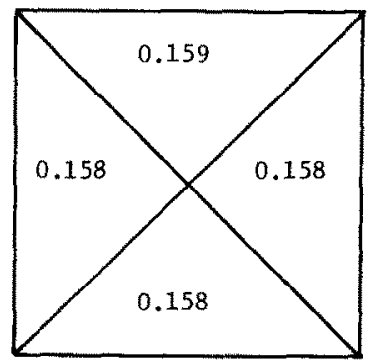

Element B

Fig. 10. Pressure distribution in quadrilaterals for a rigid punch problem. 


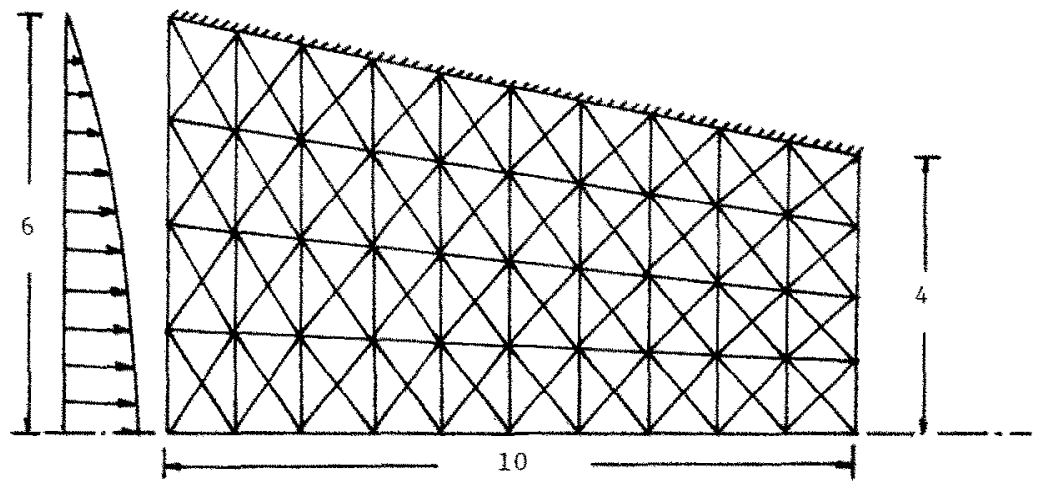

Fig. 11. Finite element model of the inclined pipe fow.

deformation analysis of elasto/visco-plasticity. To do this, we solve the Stokes flow problem both by the 4CST element and by that slight variant of the 4CST element, obtained by perturbing the location of the center node in a quadrilateral. If the center node is identified with the centroid of a quadrilateral, the numerical results for the problem shown in Fig. 11 become as in Fig. 12. If the average value of the pressure field is involved, both meshes
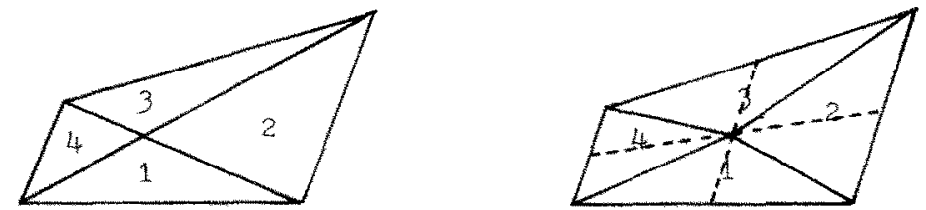

$a_{1}+a_{3}=a_{2}+a_{4}$

$$
a_{1}+a_{3} \neq a_{2}+a_{2}
$$
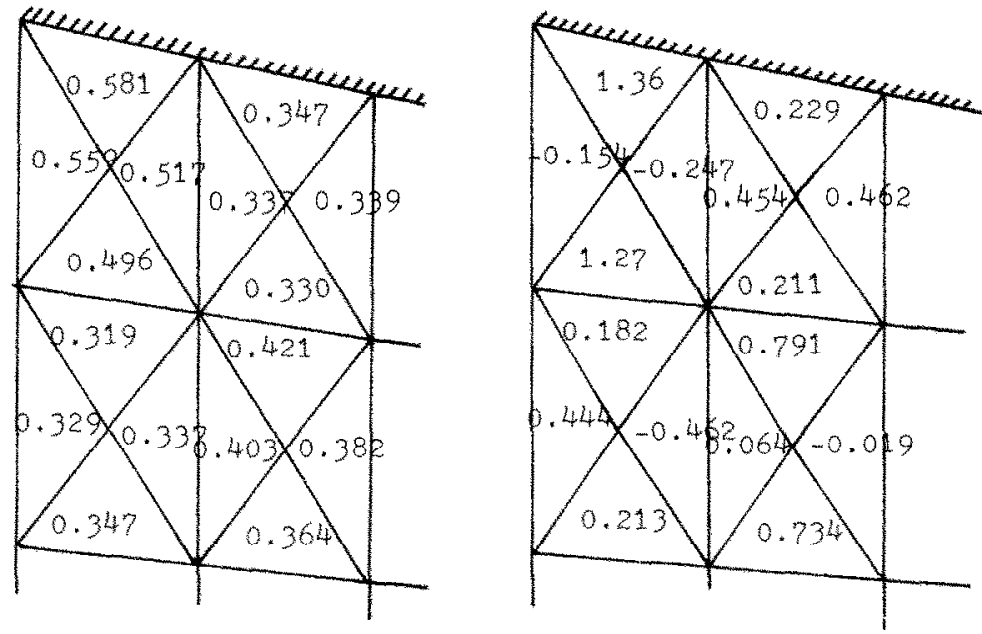

\begin{tabular}{|c|c|c|c|c|}
\hline $\begin{array}{l}\text { average pressure } \\
\text { (no Filtering) }\end{array}$ & $\begin{array}{l}0.539 \\
0.333\end{array}$ & $\begin{array}{l}0.339 \\
0.393\end{array}$ & $\begin{array}{l}0.557 \\
0.325\end{array}$ & $\begin{array}{l}0.339 \\
0.402\end{array}$ \\
\hline & \multicolumn{2}{|c|}{-0.065} & \multicolumn{2}{|c|}{-0.0738} \\
\hline $\begin{array}{l}\text { average pressure } \\
\text { (filtering on) }\end{array}$ & $\begin{array}{l}0.474 \\
0.398\end{array}$ & $\begin{array}{l}0.404 \\
0.328\end{array}$ & $\begin{array}{l}0.483 \\
0.399\end{array}$ & $\begin{array}{l}0.413 \\
0.328\end{array}$ \\
\hline
\end{tabular}

Fig. 12. Pressure distributions for two different 4CST finite elements for incompressible materials. 
provide almost the same results. However, if an individual triangular element is considered, the two meshes yield entirely different pressure fields. If the yielding of materials is checked in each triangle, the results may be very different from the ones by the average schemes for both meshes. This occurs in large deformation analyses by use of the updating process of the finite element mesh. The stress field is very sensitive to the location of the center node of a quadrilateral. This property might be important for the study of unstable phenomena like the necking of a bar in the tension test.

The final remark on the 4CST element relates to smoothing the local oscillation observed in

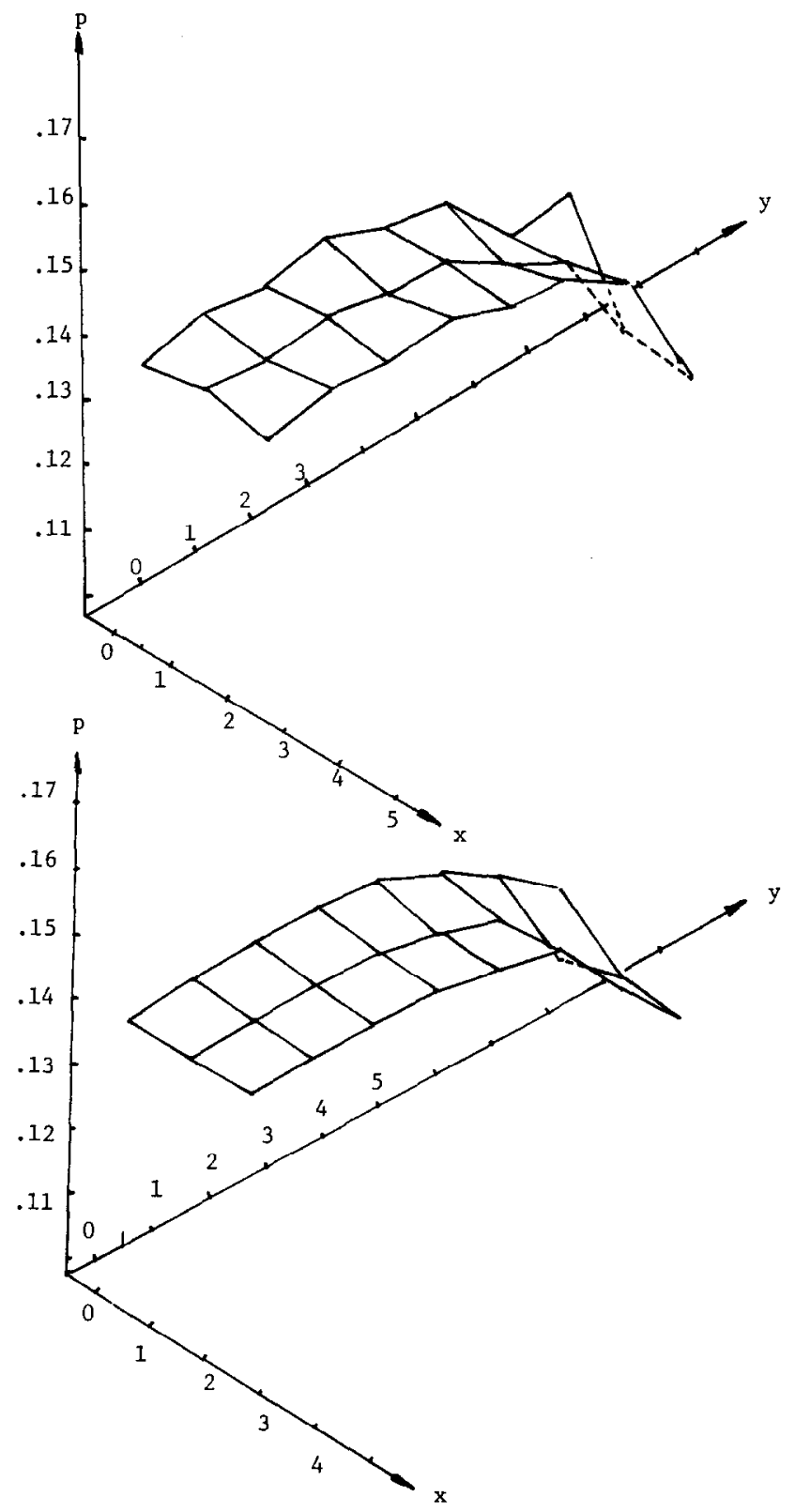

Fig. 13. Pressure fields with and without the filtering scheme for rigid punch problem. 
the pressure distribution. It is interesting that the local oscillation in the pressure has quite a similar pattern to the global kernel $K_{h}^{*}$ of the approximate gradient operator $B_{h}^{*}$. Noting the fact that the 4CST-element is equivalent to the 4-node isoparametric element with the reduced integration for the penalty term $\left(q_{h}\right.$, div $\left.v_{h}\right)$ after taking the average of the pressure, the local oscillation in the pressure can de reduced by the filtering scheme

$$
\begin{aligned}
& \tilde{p}_{\mathrm{I}}=p_{\mathrm{I}}-C, \quad \tilde{p}_{\mathrm{II}}=p_{\mathrm{II}}+C, \\
& \tilde{p}_{\mathrm{III}}=p_{\mathrm{II}}-C, \quad \tilde{p}_{\mathrm{IV}}=p_{\mathrm{IV}}+C, \quad C=\frac{1}{4}\left(p_{\mathrm{I}}-p_{\mathrm{II}}+p_{\mathrm{II}}-p_{\mathrm{IV}}\right)
\end{aligned}
$$

as shown in [14] and in [8] by a different analysis. In the analysis of convergence of the penalty finite element approximation with 4-node isoparametric element, it was necessary for the convergence to impose $\frac{1}{4}\left(\tilde{p}_{1}-\tilde{p}_{\mathrm{II}}+\tilde{p}_{\mathrm{III}}-\tilde{p}_{\mathrm{IV}}\right)=0$ for the pressure $\tilde{p}^{h}$ in [14]. This presents the filtering scheme (22) to the penalty method as a post-processing scheme to obtain a smooth pressure distribution. In Fig. 13, we show two pressure distributions for the rigid punch problem. It is clear that the filtering scheme (22) provides a smoother distribution of the pressure. As discussed above, the deformation (or the velocity field) has similar local oscillation. Thus, if the 4CST element is to be applied to solve Navier-Stokes flow problems with an incompressibility assumption by using penalty methods, one suggestion is that the velocity field to evaluate the convection term $u_{j} \partial u_{i} / \partial x_{j}$ be defined as its average value in each quadrilateral instead of in each triangle in order to obtain smoother results.

\section{Acknowledgment}

The author expresses his sincere thanks to Professor $\mathbf{J}$. Tinsley Oden for his discussions on the subject of the penalty method. During the work, the author was partially supported by NSF, G-MEA-8109221.

\section{References}

[1] J.C. Nagtegall, D.M. Parks and J.R. Rice, On numerically accurate finite element solutions in the fully plastic range, Comput. Meths. Appl. Mech. Engrg. 4 (1974) 153-177.

[2] O.C. Zienkiewicz, Constrained variational principles and penalty analysis function methods in finite elements, Lecture Notes on Mathematics Differential Equations, Vol. 203 (Springer, Berlin, 1973).

[3] O.C. Zienkiewicz, R.L. Taylor and J.M. Too, Reduced integration techniques in general analysis of plates and shells, Internat. J. Numer. Meths. Engrg. 3 (1971) 275-290.

[4] D.S. Malkus and T.J.R. Hughes, Mixed finite element methods-reduced and selective integration techniques: a unification of concepts, Comput. Meths. Appl. Mech. Engrg. 15 (1978) 63-81.

[5] J.N. Reddy, On the accuracy and existence of solutions to primitive variable models of viscous incompressible fluids, Internat. J. Engrg. Sci. 16 (1978) 921-929.

[6] M. Bercovier, Perturbation of mixed variational problems. Application to mixed finite element methods, Rev. Francaise Automat. Informat. Recherche Opérationelle Anal. Numér. 12 (1978) 211-236.

[7] J.T. Oden and N. Kikuchi, Finite element methods for constrained problems in elasticity, Internat. J. Numer. Meths. Engrg. 18 (1982) $701-725$. 
[8] R.L. Lee, P.M. Gresho and R.L. Sani, Smoothing techniques for certain primitive variables solutions of the Navier-Stokes equations, Internat. J. Numer. Meths. Engrg. 14 (1979) 1785-1804.

[9] R. Temam, Navier-Stokes Equations (North-Holland, Amsterdam, 1977).

[10] B. Mercier, Topics in finite element solution of elliptic problems, TATA Institute of Fundamental Research, Bombay, 1979.

[11] J.P. Aubin, Approximation of Elliptic Boundary-Value Problems (Wiley, New York, 1972).

[12] J.L. Lions, Quelques Méthodes de Résolution de Problèmes aux limites Nonlinéaires (Dunod, Paris, 1969).

[13] B. Mercier, A conforming finite element method for two-dimensional incompressible elasticity, Internat. J. Numer. Meths. Engrg. 14 (1979) 942-945.

[14] Y.J. Song, J.T. Oden and N. Kikuchi, Discrete LBB-conditions for RIP-finite element methods, TICOM Report 80-7, The University of Texas at Austin, 1980.

[15] R. Courant, Calculus of variations and supplementary notes and exercises; Rev. ed. amended by J. Moser (University Press, New York, 1956). 